\title{
The Buying Impulse and Perceptions of the Physical Self
}

\author{
Michael Minor, Tofazzal Hossain \\ Robert C. Vackar College of Business \& Entrepreneurship, University of Texas, Edinburg, TX, USA \\ Email:michael.minor@utrgv.edu, tofazzal.hossain01@utrgv.edu
}

How to cite this paper: Minor, $M$. and Hossain, T. (2017) The Buying Impulse and Perceptions of the Physical Self. Theoretical Economics Letters, 7, 1899-1924. https://doi.org/10.4236/tel.2017.77129

Received: October 23, 2017

Accepted: November 19, 2017

Published: November 22, 2017

Copyright $(2017$ by authors and Scientific Research Publishing Inc. This work is licensed under the Creative Commons Attribution International License (CC BY 4.0).

http://creativecommons.org/licenses/by/4.0/ (c) (i) Open Access

\begin{abstract}
General trait-based approaches to the study of impulsive buying fail to explain the product-specific nature of this behavior and the relevance of specific self-motives in this context. Two related studies were conducted to understand the role of domain-specific physical self-perceptions on the context dependent nature of impulsive buying. The first study showed that physical self-perceptions are better predictors of context-specific impulsive buying than global self-measures. The second study grouped people with high and low physical self-esteem (PSE) to understand the role of PSE on product-specific, impulsive buying tendencies. Results showed that the impulsive buying tendency of individuals toward different products changes as a function of the relevance of product to physical self-perceptions. It was also found that perceived importance in the physical domain (PIP) may be an underlying factor. Overall, current research suggests impulsive buying is a product-specific behavior such that physical self-images along with perceived importance attributed to these images may affect individuals' impulsive buying tendencies, depending on the self-related function of products.
\end{abstract}

\section{Keywords}

Impulsive Buying, Physical Self-Perceptions, Self-Esteem, General Impulsivity

\section{Introduction}

Many economists and social-cognitive psychologists consider economic behaviors as purely rational, carefully planned, and intentional activities [1]. In economics, for example, the concept of "homo economicus" theorizes that people are rational market consumers with the ability to make stable economic judgments to maximize their savings and profits in marketing activities [2] [3]. Well known socio-cognitive behavior models (i.e., theory of reasoned action and theory of planned behavior) also suggest that people engage in considerable cognition such as considering behavioral intentions along with perceived personal and social norms before engaging in 
any social behavior [4] [5]. Although these well-established rational choice behavior models can account for many economic behaviors in which people search for the lowest prices, compare products, and make careful decisions before purchasing goods or making investments, they fail to explain the globally recognized economic behavior of impulsive buying.

In today's market place, unplanned and immediate purchases have been widely considered as pervasive and distinctive characteristics of consumers' buying behavior [6] [7] [8] [9]. In 1977, for example, the Point of Purchase Advertising Institute's 8th DuPont Consumer Buying Habits Study indicated the prevalence of unplanned purchases among American consumers when it declared that, "nearly 66\% of every dollar spent results from some decision were made in the store". The validity of this observation is clearly sup-ported by a recent CreditCards.com poll in which 5 of 6 Americans reported making impulse purchases often [10]. Recent marketing research on unplanned purchasing has also shown that buying on impulse can account for $40 \%$ to $80 \%$ of all purchases, depending on the product category [11] [12] [13] [14] [15]. Recognition of the growing trend of impulsive buying across all segments of society has prompted consumer researchers to begin focusing on understanding the behavioral antecedents, correlations, and consequences of impulsive buying, and they are doing so from a wide spectrum of theoretical perspectives and research approaches [6] [16]-[21].

Impulsive buying was once commonly defined as "an unplanned purchase" [22] [23]. Stern [24] enriched this definition by suggesting that planned, pure, reminder and suggestion could be considered four major types of impulse purchasing (p. 59). Adding a fifth dimension, "without regard for the consequences", Rook [22] further deepened the definition of impulsive buying as, "a sudden, powerful and persistent urge to buy some-thing immediately" (p. 191). Unlike planned purchasing behavior, impulsive buying is, "less deliberate and more arousing and irresistible" (p. 164) [23]. Due to its importance, impulsive buying has been receiving increased attention from researchers, especially those in consumer behavior and retailing re-search.

Initial studies on impulse buying were conducted primarily for retailing purposes [13] [25] [26] [27]. The main motivation was to categorize products, based on product characteristics, as impulsive or non-impulsive items. This product-based marketing led retailers to redesign shopping environments to maximize customers' exposure to impulsive products. They reorganized in-store display stands and promotions so as to increase point of purchase sales [24] [28] [29]. Business scholars, however, criticized this early retailing approach for having definitional and conceptual limitations. From the definitional standpoint, impulse buying behavior was simply regarded as an unplanned purchase for only certain types of items, whereas the conceptual focus was limited to the way in which products were offered to consumers (i.e., physical proximity, appearance, and presentation) without considering individual consumer characteristics. Later research, however, has consistently shown that almost any type of product can be bought on impulse, 
and not all unplanned purchases can be considered as impulse buying [30] [31].

Consequently, many researchers have come to agree that impulsive buying is a complex behavioral phenomenon that depends on various personality characteristics, inner psychological states and social-environmental settings, for instance [6] [20] [32] [33]. In his seminal paper, Rock [22] touched upon most of these aspects and defined impulsive buying as, "sudden, often powerful and persistent urge to buy something immediately". He also emphasized the psycho-emotional aspects of impulsive buying with pre-behavior "hedonistic motives" and post-behavior "emotional conflicts" (p. 191). In addition to these psycho- emotional attributes, a wide set of socio-demographic characteristics including age [34], gender [18], and culture [23] have been recognized as predictors of impulse buying. Several studies have extensively studied a variety of cognitive characteristics ranging from self-related concepts (i.e., self-esteem or self-control) to personality traits (i.e., general impulsiveness, identity concerns, or a narcissistic personality) to explain the psychological underlying mechanisms of impulsive buying [35]-[40].

Although this large body of research significantly contributed to our understanding of impulsive buying behavior, inconsistent results over time confounded conceptualizations for the role of certain psychological constructs in impulsive buying [20]. Both positive and negative feelings, for example, are found to be significant predictors of impulse buying [21] [41]. With respect to personality factors and using an individual's relatively stable and general tendency to impulsivity, previous researchers relied primarily on psychological trait models to explain impulsive buying [7]. Because they have general impulsivity as a personality trait, individuals are assumed to be potential impulsive buyers for all product categories [27]. This understanding, however, fails to account for recently reported findings that specific products (such as fashionable clothing) are consistently purchased impulsively, while other products (such as housing consumables) are not [36].

Self-esteem and self-control mechanisms are also two recently centralized psycho-logical constructs in research on impulsive buying [36] [39] [42] [43] [44]. Although impulsive buying has generally been linked to low self-esteem, threatened self-views, and the negative effects associated with damaged self-worth, underlying motivations of these relationships are still unclear and under debate [17] [42]. The promising arguments for the relationship between distorted self-views and excessive buying are provided by the theory of symbolic self-completion (SCT) [45]. Briefly, SCT contends that impulse buying is employed as a compensatory action to avoid negative feelings associated with low self-esteem and to restore one's self-status through identity-relevant possessions. When individuals perceive a discrepancy between their ideals and perceived self-images, they may tend to seek compensatory possessions and may invest their economic resources in acquiring these self-image related possessions for self-presentational motives in an impulsive manner [46]. In a series of connected studies, Dittmar and colleagues provided convincing evidence that self-related psychological functions of impulsive consuming are consistent with symbolic self-completion theory [33] [36] [42] [43]. 
In one of their in-depth qualitative studies, Dittmar and Drury [36] reported that consumers who have high self-discrepancies are more likely to buy on impulse and are motivated mainly by self-related reasons. While these studies extend our preliminary understanding of the self-related mechanisms that motivate impulsive buying, they are primarily limited to general impulsive buying tendencies, and, thus, do not address the context-specific nature of impulsive buying in relation to self-related motives. Moreover, previous research on consumer behavior treated the self-system as a unidimensional psychological construct, and it focused exclusively on the link between global self-esteem and impulsive buying tendencies [36] [39] [44]. Today, many social psychologists have recognized the multi-dimensional nature of the self-system and convincingly showed that domain specific self-perceptions such as academic, emotional and physical self-esteem are better predictors of context-specific behaviors than global self-beliefs. One important aspect of this multi-dimensional self- structure is physical self-esteem because of its strong influence on global self- worth and sense of individual integrity [47]. A variety of studies have documented the proposition that physical self-perceptions are associated with exercise behavior in all age groups, adherence to rehabilitation in patient populations, eating disorders, social anxiety among women, depression, psychological well-being, and self-presentational concerns in the general population [47] [48] [49] [50] [51].

Given the close connections between self-presentational concerns and impulsive buying tendencies, individual perceptions in the multi-dimensional structure of the physical self-system may also provide valuable insights into the complex nature of impulsive buying. To date, however, no systematic research has been conducted to understand the role of domain-specific physical self-perceptions on the product-specific nature of impulsive buying tendencies. Moreover, the effects of well-established personality concepts in consumer research, concepts such as self-control and general impulsivity, have not been controlled for the role of physical self-perceptions in impulsive buying behavior.

Therefore, this article examines the relationships between physical self-perceptions and the product-specific nature of impulsive buying after controlling for the effects of the degree of self-control and general impulsivity. Three main assumptions are hypothesized and tested by two separate studies. First, we assume impulse buying behavior can be better explained by specified psychological characteristics (i.e., physical self-perceptions) rather than by general trait measures (global self-esteem). Second, we believe the relationship between physical self-perceptions and impulsive buying is independent of the effects of self-control and general impulsivity. Third, we propose that the relevance of the product type to physical self-motives may change the nature of impulsive buying tendencies. Following this introduction, we briefly discuss our theoretical foundation. After that, we test our first two assumptions through study 1 and investigate our last assumption in study 2 . We then present our results. Lastly, we discuss our findings and contributions. 


\section{Theoretical Background}

\subsection{Multidimensional Self System and the Physical Self Esteem}

During the past 40 years, a large body of research in social psychology has provided important empirical findings on the nature of our self-system. One essential point has been the recognition that the self-system is constructed out of multiple domain specific selves and organized in a hierarchical manner. Within this multidimensional and hierarchical conceptualization of the self-system, global self-esteem (GSE) is placed at the apex of the hierarchy [52] which is then divided into specific domains such as the academic, social, emotional, and physical self (Figure 1). In this framework, positive evaluations, self-sub-domains are assumed to improve GSE [53] [54]. Each sub-domain is also proposed to organize in a multi-dimensional structure with domain specific perceptions such that, for example, evaluations and feelings about physical health, body fat and appearance contribute to our physical self-esteem (PSE) (Figure 1).

Although social psychological research has shown that perceived efficacy in these lower order self domains (such as sub-domains of physical self-esteem) is a better predictor of actual behaviors than global self domains (i.e. global self-esteem), impulsive buying studies in consumer and marketing literature have exclusively focused on the role of GSE in relation to buying preferences. Domain specific physical self-perceptions, however, have been considered as the primary component of our global self-system and evaluations in the physical domain are assumed to be the essential underlying motives of many social behaviors [55].

\subsection{The Physical Body and the Self}

A growing body of literature shows the essential role of bodily perceptions in the construction of our self-identities in today's contemporary cultures. In their seminal studies Shilling [56] and Giddens [57] have clearly pointed out the ultimate

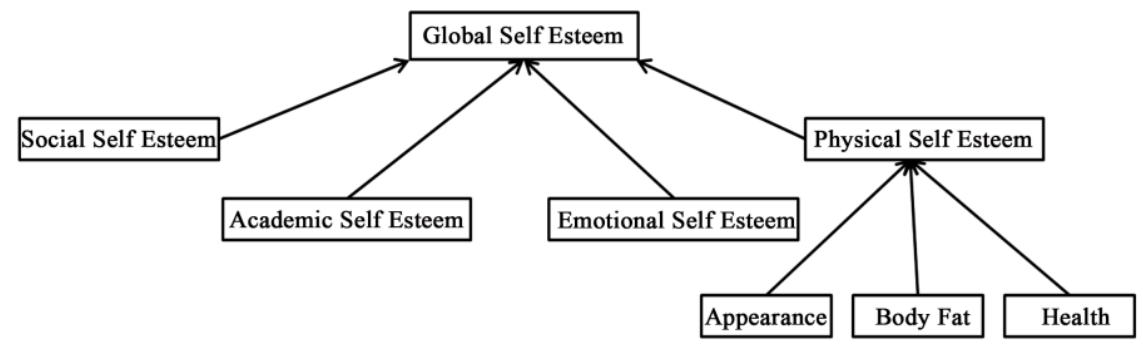

Figure 1. Hierarchical and multi-dimensional self-esteem model.

connections between the self and the physical body throughout the life span. Synnott [58] considers the physical body as the prime symbol and determinant of our selves. Prominent self-researchers also suggest that developmental roots of the self-system originate from the physical body in the sense that first notions about the self are based on the physical self during childhood years [59]. According to Baumeister [60], for example, learning to distinguish one's own body from the rest of the environment is the first step in the formation of "self-concept" in early 
childhood. During adolescent and adulthood periods, the physical body along with its abilities and attributes, such as its shape, size, appearance and content not only reflect personal idiosyncrasies but are also indicative of certain socio-demographic characteristics, such as social class, health, success and wealth [61]. Kirk [61] argues that the presentation of the physical body as a social symbol, critical sign of success and personal worthiness are becoming especially salient in contemporary western societies due to changes in the psychological meaning and functions of consuming. In today's consumer culture, the body is no longer considered just a physical entity with its physiological functions but an important consumer good with its own exchange value if it adopts the social norms of physical beauty and attractiveness. This understanding suggests a new type of relationship between the physical body and the self, in which individuals are excessively self-conscious about physical changes in their bodies, fighting against aging, constantly searching for signs of physical deformation and are more sensitive than ever to evaluations about their physical characteristics [61]. A typical person in the 21 st century tends to invest more time, effort and financial sources on monitoring and controlling his/her physical appearance and develops long-term behavioral strategies to be able to be in line with the cultural standards of physical beauty. It is therefore not surprising that dieting and regular exercise regimens are essential long-term behavioral strategies for transforming body size and shape in accordance with cultural norms, whereas fashionable clothing, accessories, cosmetic products and cosmetic surgery increasingly become important segments of today's consumer market.

Given the changing nature of social meaning of the physical body in our consumer culture, body related perceptions have now become core elements of individuals' self-integrity. Indeed, research has shown that physical self-perceptions (PSPs), for example, are substantially associated with the global sense of self-esteem. People who think they look physically attractive or get positive evaluations from others with respect to their physical appearance have higher general self-esteem than people who are not happy or satisfied with how they look or are negatively evaluated on their physical appearance. Thus, feelings and perceptions in the physical domain have been considered as crucial aspects in the multidimensional and hierarchical model of the self-system [47]. Accordingly, physical self-evaluations are widely and frequently investigated psychological constructs in a variety of contexts. It has been, for example, documented that the physical self is associated with a variety of health-related behaviors, including exercise maintenance and dieting, as well as various other psychological well-being indicators such as social anxiety, depression and self-presentational concerns [50]. It is, therefore, quite reasonable to assume that such an essential psychological construct should also play an important role in individuals' buying habits/preferences, and specifically affect individuals' impulsive buying tendencies as an underlying motive, depending on the buying context.

\subsection{The Current Study}


In the consumer psychology literature, it is now well acknowledged that impulsive buying behavior is primarily driven by certain personality characteristics, such as general impulsivity, or various psychological needs, ranging from hedonistic or positive feelings to negative mood states, rather than physical necessities. These studies regarded impulse buying as a general and relatively stable consumer trait that is associated with general impulsivity, low self-control, and lack of deliberation on the cognitive dimension [20]. On the affective dimension, it was also reported to be associated with the need for immediate gratification, excitement, and avoidance of negative feelings [20]. Thus, the traditional approach based on trait theories mainly assume that consumers having certain psychological profiles, as explained above, should also have a general tendency to purchase any type of product on impulse. However, this research fails to explain the context specific nature of impulsive buying behavior and, especially, the relevance of this context to domain specific self-motives. Although Dittmar and Drury [36] showed that discrepancies between ideal and perceived selves drive people to engage in impulsive buying for certain products to compensate for perceived self-discrepancies, they treated the self-system as a unidimensional construct and, thus, did not specify the multidimensional aspects of the perceived self-discrepancies. Consequently, how the specific aspects of the self-system, such as the PSPs, are related to impulsive buying in a product specific manner is largely unknown. Therefore, based on the discussions above, we hypothesize that:

Hypothesis 1: Domain specific physical self-perceptions will be better predictors of impulsive buying tendencies than global self-perceptions.

The research from [18] [36] also primarily relied on symbolic self-completion theory which mainly posits that many products may have symbolic meanings for reflecting individuals' identities, personal values, social status and life styles. Purchasing these products may, thus, have self-serving functions in a way that they can help to define and reflect personal identities, and verify selves. The theory also proposes that self-affirmation based assumptions especially becomes salient when perceived inadequacies threaten or compromise current self-images and thus motivate people to purchase products that represent threatened or compromised aspects of the self for compensation motives [44]. This explanation, however, seems only suitable for "reactive" strategies where the ideal self-image is already wounded or still not attained. However, the social psychological literature has shown that people not only display "reactive" behaviors with self-enhancement motives in response to threatened self-images or increased self-discrepancies, but also "pro-actively" employ preventive strategies with self-protection motives to deal with future situations that can distort current self-images [60]. Within the context of buying, for example, people with high physical self-esteem may tend to spend more money on fashion clothes with the intention of exhibiting their attractiveness to garner positive evaluations (Self-enhancement) and, thus, may engage more in impulsive buying depending on the product context. On the other hand, they may avoid buying snack foods or binge eating since excessive caloric consumption may distort their current physical appearance and increase the discrepancy between ideal and actual physical 
self-images. Although these propositions intuitively sound reasonable, empirical conclusions have yet to be observed in the impulsive buying literature.

The current literature proposes that specific self-perceptions in the physical domain, such as perceived physical health, physical appearance, body fat and physical self-esteem will be related to impulsive buying tendencies depending on the context of buying, such as whether the product has relevance to physical self-perceptions or not. Taken together, the literature led to the development of the following two hypotheses:

Hypothesis 2: The role of physical self-perceptions on impulsive buying tendencies will be independent of the effects of self-control and impulsivity levels.

Hypothesis 3: Impulsive buying intentions of individuals will change as a function of the relevance of the product to physical self-motives.

In order to test the hypotheses, we conducted two related studies to understand the role of domain specific physical self-perceptions on the context dependent nature of impulsive buying. Study 1 was conducted to test the assumptions mentioned in Hypothesis 1 and Hypothesis 2. Then we conducted study 2 to test the nature of impulsive buying tendency depending on the relevance of the product type to physical self-motives, which proposed in our Hypothesis 3. In the next section, we present the sampling methodology, survey design, data collection and analysis as well as results and discussion for both study 1 and study 2 .

\section{Study 1}

This study was designed to explore relationships between domain specific selfperceptions and product specific impulsive buying tendencies. The main argument underlying the current design is to study whether: 1) impulse buying behavior can be better explained by specified psychological characteristics (i.e. physical self-perceptions) rather than general trait measures (global self-esteem); and 2) the relationship between physical self-perceptions and impulsive buying tendencies is independent of the effects of self-control and general impulsivity. As a preliminary step, therefore, it was hypothesized that domain specific physical self-perceptions, but not global self-esteem, are significantly correlated with product-specific impulsive buying tendencies. In the context of impulsive buying, physical self-perceptions should predict product-specific impulsive buying tendencies better than global measures of self-esteem. In previous research, for example, Jones and colleagues [62] showed that product-specific measures of impulse buying tendencies are highly correlated to actual impulsive purchasing behavior when compared to general impulsivity. However, the role of domain specific self-perceptions, as compared to the global self, on product specific impulsive buying is not examined.

\subsection{Methods}

Participants were 198 undergraduate business-major students (90 males with Mage 
$=22.02, \mathrm{SD}=3.03$ and 108 females with Mage $=21.47, \mathrm{SD}=2.63$ ) attending a large US university in the Houston metropolitan area. All participants were offered partial course credit for their participation in the study. At the beginning of the study, informed consent forms were distributed describing the general nature of the study's procedures. The voluntary and confidential nature of the study was emphasized, and students were asked, "not" to write their names on any of the questionnaires. Questionnaires were coded with unique ID numbers in advance.

Global self-esteem was measured by using the Rosenberg self-esteem scale (RSE) [63] which is one of the most widely used instruments for measuring global self-esteem in research settings. RSE consists of 10 items on a 4-point Likert type scale ranging from "strongly agree" to "strongly disagree". The scale has five positively worded (e.g., "On the whole, I am satisfied with myself”), and five negatively worded items (e.g., "I certainly feel useless at times"). For this study, internal consistency of the overall scale was 0.89. The Physical Self-Description Questionnaire (PSDQ) [64] was used to measure domain specific physical perceptions of participants. The scale originally consists of 70 items designed to measure nine specific subscales of the physical self-concept (health, coordination, physical activity level, body fat, sport, appearance, strength, flexibility and endurance), as well as a general component of physical self-concept and global self-esteem. Each subscale is represented by 6 or 8 items; each item in these scales is a simple declarative statement and participants respond using a 5-point true-false response scale. 3 subscales of the PSDQ measuring "perceived appearance" (e.g., "I am better looking than most of my friends."), "physical health" (e.g., "I have to go to the doctor because of illness more than most people my age."), "body fat" (e.g., "My waist is too large."), and a general component of "physical self-esteem" (e.g., "I am satisfied with the kind of person I am physically") were used in this study. Each subscale has acceptable internal consistency levels including 0.79 for physical appearance, 0.82 for physical health, 0.85 for body fat and 0.89 for general physical self-esteem. Self-control was measured with the Brief Self-Control Scale developed by Tangney and colleagues [65]. The self-control scale originally consisted of 36 items to measure individuals' perceived self-control over their thoughts (e.g., "I have trouble concentrating"), emotions (e.g., "I say inappropriate things"), impulses (e.g., "I am good at resisting temptation"), performance regulation (e.g., "I am able to work effectively toward long-term goals"), and habits (e.g., "I have a hard time breaking bad habits"). The brief version of the self-control scale is reported to be highly correlated with $(0.93)$ the original scale, thus covering the same content [65]. The brief self-control scale consists of 13 items on a 5-point Likert type scale ranging from "not at all like me" to "very much like me". Internal consistency of the scale was 0.88 for this study.

Impulsiveness was measured by using the "Impulsivity (or Lack of Control)" sub-scale of the Multidimensional Personality Questionnaire-MPQ [66]. MPQ was originally developed to quantify primary personality dimensions ranging from wellbeing to aggression. The Impulsivity (or Lack of Control) subscale focuses on 
the degree to which individuals have control over spontaneous, reckless and careless behaviors. The impulsivity sub-scale consists of 24 items and participants were asked to indicate if each item (e.g., "People consider me a rather freewheeling and spontaneous person") is a "True" or "False" description of themselves on a 2-point scale.

Finally, both general impulsive buying and product specific impulsive buying tendencies were measured by using Rook and Fisher's [9] 9-item buying impulsiveness scale (e.g., "Sometimes I feel like buying things on the spur-of-the-moment."). The wording of the scale was altered appropriately to assess the impulsive buying tendency toward specific products including clothing (e.g., "Sometimes I feel like buying my clothes on the spur-of-the-moment."), and snack food (e.g., "Sometimes I feel like buying snack food-chocolate, candies or chips-on the spur-of-themoment."). The items were rated on a 5-point Likert type scale ranging from "strongly agree" to "strongly disagree".

\subsection{Results}

Descriptive (mean and standard deviations) characteristics of all measures are provided in Table 1 both for men and women. Preliminary analyses were performed by using independent t-tests to identify possible gender differences both for independent (psychological measures) and dependent variables (impulsive buying tendencies). Among the independent variables, on average, women reported higher perceived body-fat $(t(196)=4.53, p<0.05)$ as compared to men with a medium effect size $(r=0.30)$. In regard to the impulsive buying variables, women also reported having significantly higher general impulsive $(t(196)=4.56$, $p<0.05)$, clothing impulsive $(t(196)=3.13, p<0.05)$ and snack-food impulsive $(t(196)=3.39, p<0.05)$ buying tendencies when compared to men, with medium $(r=0.31)$, small $(r=0.22)$, and small $(r=0.23)$ effect sizes respectively. In both groups, clothing impulsive buying was significantly higher than all other types of impulsive buying tendencies $(p<0.05)$ with moderate effect sizes $(r>0.30)$.

Initial relationships among study variables were identified by bivariate Pearson Table 1. Mean and standard deviations of all measures.

\begin{tabular}{ccccc}
\hline & \multicolumn{2}{c}{ Men } & \multicolumn{2}{c}{ Women } \\
\cline { 2 - 5 } & Mean & Std & Mean & Std \\
\hline Psychological Variables & & & & 3.55 \\
Global Self Esteem & 31.86 & 3.43 & 30.89 & 4.73 \\
Physical Self Esteem & 26.48 & 4.87 & 25.32 & 4.82 \\
Perceived Appearance & 25.03 & 4.64 & 24.00 & 5.20 \\
Perceived Body Fat & 17.74 & 4.44 & 20.89 & 3.36 \\
Perceived Health & 17.25 & 3.19 & 17.36 & 8.74 \\
Self-Control & 40.41 & 9.28 & 38.41 & 3.82 \\
Impulsiveness & 10.44 & 3.60 & 09.94 & \\
\hline
\end{tabular}




\section{Buying Tendencies}

General Impulsive

Clothing Impulsive

Snack Food Impulsive
23.03

26.46

22.74
4.30

4.16

4.16
26.04

28.59

24.89
4.87

5.18

4.67

correlations and presented in Table 2. The highest positive correlations were achieved among general impulsive buying and context specific impulsive buying tendencies, indicating that people with impulsive buying characteristics are also more frequent impulsive buyers in different contexts.

GSE was found to be positively associated with PSE $(r=0.22, p<0.01)$, appearance $(r=0.26, p<0.01)$ and negatively related with body fat $(r=-0.21, p<$ $0.01)$. Significantly negative but weak correlations were also found between GSE and general impulsive buying tendency $(r=-0.14, p<0.05)$ indicating that those with low global self-esteem tend to be more impulse buyers in general. No significant correlations, however, were found between global self-esteem and context specific impulsive buying tendencies (Table 2). PSE, on the other hand, were found to be significantly, but differentially, correlated with context specific impulsive buying tendencies. Results indicated that PSE is positively related with impulsive clothing buying $(r=0.33, p<0.01)$, but negatively with impulsive snack food buying $(r=-0.23, p<0.01)$ tendencies suggesting the context-specific role of physical self-perceptions on impulsive buying behavior. Appearance was also found to be negatively, but weakly, associated with impulsive snack food buying $(r=-0.15, p<0.05)$. Here those results partially supported our hypothesis 1 , which indicated that PSE could better explain the impulse buying behavior than general trait measures such as GSE. However, based on different types of products, PSE may explain impulse buying behavior differentially.

To better understand the relationships between self-perceptions and impulsive buying tendencies a series of hierarchical multiple regression analyses were conducted. Self-control and impulsivity scores were entered in a regression Table 2. Bivariate relationships among self perceptions, impulsive buying tendencies and control variables $(* p<0.05, * * p<0.01)$.

\begin{tabular}{|c|c|c|c|c|c|c|c|c|c|c|}
\hline & 1 & 2 & 3 & 4 & 5 & 6 & 7 & 8 & 9 & 10 \\
\hline 1-GSE & & $0.217^{* *}$ & $0.260^{* *}$ & $-0.209^{* *}$ & 0.054 & 0.012 & -0.057 & $-0.143^{*}$ & 0.052 & -0.053 \\
\hline 2-PSE & & & $0.405^{* *}$ & $-0.206^{* *}$ & -0.001 & 0.099 & $-0.180^{*}$ & -0.073 & $0.328 * *$ & $-0.226^{* *}$ \\
\hline 3-Appearance & & & & $-0.385^{* *}$ & -0.013 & 0.087 & -0.168 & -0.084 & 0.099 & $-0.147^{*}$ \\
\hline 4-Body Fat & & & & & -0.004 & -0.002 & 0.035 & $0.151^{*}$ & -0.018 & 0.114 \\
\hline 5-P. Health & & & & & & 0.093 & -0.075 & -0.037 & 0.023 & -0.058 \\
\hline 6-Self Control & & & & & & & $-0.461 * *$ & -0.067 & 0.017 & 0.014 \\
\hline 7-Impulsivity & & & & & & & & $0.167 *$ & -0.096 & -0.093 \\
\hline 8-Gen. IBT & & & & & & & & & $0.517 * *$ & $0.683^{* *} *$ \\
\hline 9-Cloth IBT & & & & & & & & & & $0.394 * *$ \\
\hline 10-Snack IBT & & & & & & & & & & \\
\hline
\end{tabular}


model at the first step to control their possible confounding effects for the predictive role of self-perceptions on impulsive buying tendencies. All self-perception variables (GSE, PSE, Appearance and Body-fat) were entered into the model at the second step as independent predictors for impulsive buying tendencies. Scatter plots of relationship between self-perceptions and impulsive buying tendencies are provided in Figure 2. In detail, the fit lines in Figure 2 present the bivariate correlations between physical self-perceptions and impulsive buying tendencies. Results of the hierarchical multiple regression analyses are also provided in Table 3. Regression analyses showed that control variables (self-control and impulsivity) significantly predicted general impulsive buying tendencies in the first step but accounted only for $3 \%$ of variability $\left(\mathrm{R}^{2}=0.031, \mathrm{~F}=3.13, p<0.05\right)$.

Although the self-perception variables significantly improved the regression model in the second step with an additional $3 \%$ of explained variance, predictor coefficients showed that only perceived body fat scores significantly $(\beta=0.149, \mathrm{t}$ $=2.25, p<0.05$ ) contributed to the regression model (Table 3). The regression model for impulsive clothing buying tendency was significant when self-perception variables entered the model after controlling for the effects of self-control and impulsivity $\left(\mathrm{R}^{2}=0.114, \mathrm{~F}=7.08, p<0.01\right)$ with $11 \%$ of explained variance. Therefore, our hypothesis 2 is supported by showing the independent effect (11\% of variance) of physical self-perceptions on impulsive buying tendencies with the controlled effect of self-control and impulsivity levels (3\% of variance).

In addition, we found that predictor coefficients were only significant for physical self-esteem $(\beta=0.345, \mathrm{t}=4.53, p<0.01)$ indicating that individuals with higher PSE scores are more likely to be impulsive buyers for clothing. The regression model for impulsive snack-food buying tendency was also significant $\left(\mathrm{R}^{2}=\right.$ $0.078, \mathrm{~F}=2.70, p<0.05$ ) in which the only significant predictor was physical self-esteem. The direction of the relationship, however, was opposite with a 


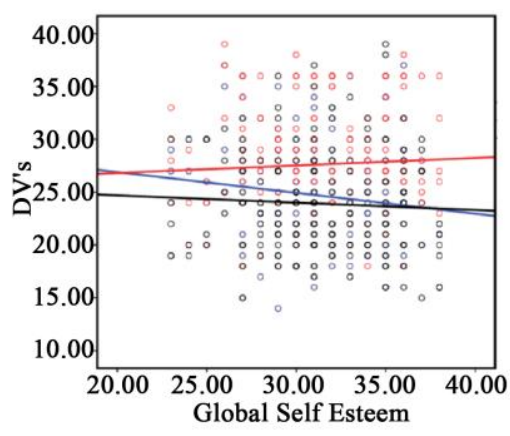

(a)

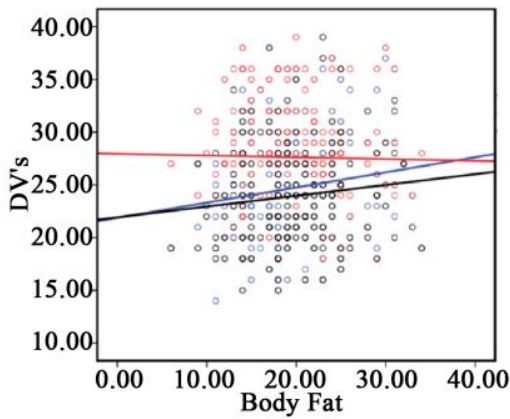

(c)

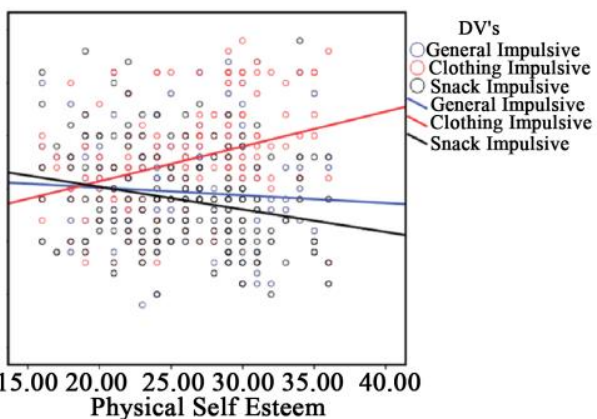

(b)

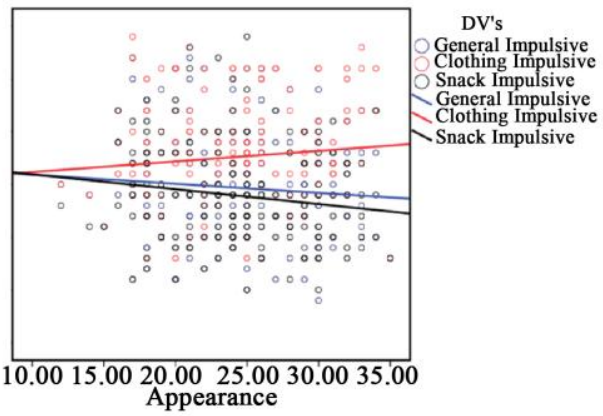

(d)

Figure 2. Scatter plots with fit lines for the bivariate correlations between physical self-perceptions and impulsive buying tendencies.

Table 3. Hierarchical regression results.

\begin{tabular}{|c|c|c|c|c|c|}
\hline General Impulsive & & & & & \\
\hline & Model 1: Self Control, Impulsivity & 0.176 & 0.031 & 3.13 & $0.04 *$ \\
\hline & Model 2: Self Perceptions & 0.236 & 0.056 & 2.49 & $0.02 *$ \\
\hline & Coefficients & $\boldsymbol{\beta}$ & $t$ & $p$ & \\
\hline & Global Self Esteem & -0.159 & -1.58 & 0.11 & \\
\hline & Physical Self Esteem & -0.039 & -0.5 & 0.61 & \\
\hline & Appearance & -0.013 & -0.16 & 0.88 & \\
\hline & Body Fat & 0.149 & 2.25 & $0.02 *$ & \\
\hline \multicolumn{6}{|l|}{ Clothing Impulsive } \\
\hline & Model 1: Self Control, Impulsivity & 0.101 & 0.01 & 1.01 & 0.37 \\
\hline & Model 2: Self Perceptions & 0.337 & 0.114 & 7.08 & $0.00 * *$ \\
\hline & Coefficients & $\boldsymbol{\beta}$ & $t$ & $p$ & \\
\hline & Global Self Esteem & -0.013 & -0.132 & 0.9 & \\
\hline & Physical Self Esteem & 0.345 & 4.53 & $0.00 * *$ & \\
\hline & Appearance & -0.028 & -0.347 & 0.73 & \\
\hline & Body Fat & 0.04 & 0.572 & 0.57 & \\
\hline \multicolumn{6}{|l|}{ Snack Impulsive } \\
\hline & Model 1: Self Control, Impulsivity & 0.099 & 0.01 & 0.96 & 0.39 \\
\hline & Model 2: Self Perceptions & 0.28 & 0.078 & 2.7 & $0.02 *$ \\
\hline & Coefficients & $\boldsymbol{\beta}$ & $t$ & $p$ & \\
\hline & Global Self Esteem & 0.017 & 0.183 & 0.85 & \\
\hline & Physical Self Esteem & -0.205 & -2.8 & $0.02 *$ & \\
\hline & Appearance & -0.064 & -0.806 & 0.42 & \\
\hline & Body Fat & 0.047 & 0.692 & 0.49 & \\
\hline
\end{tabular}


negative coefficient $(\beta=-0.205, t=-2.80, p<0.05)$ indicating that high physical self-esteem is associated with decreased impulsive buying tendency for snack-food.

\subsection{Study 1 Discussion}

This exploratory study 1 was designed to understand relationships between domain specific self-perceptions and product specific impulsive buying tendencies. Preliminary comparisons on impulse buying products revealed that clothing products, regardless of gender, are bought more on impulse than snack food, supporting the previous observations that certain product types are more likely to be the object of impulse buying than others [36]. A high level of impulse buying tendency among women is also in line with the literature confirming gender differences in impulse buying [18]. The main findings provided initial and partial support for our hypotheses that impulsive buying behavior can be better explained by domain specific psychological metrics such as physical self-percep- tions as compared to trait measures of GSE. Another unique finding of the current study is also the observation that the impulse buying tendency may change as a function of the relevance of the product context to domain-specific self-per- ceptions.

Previous marketing research has traditionally considered impulse buying tendency as a general personality trait that is primarily consistent across different product categories [7] [27]. Our correlation analyses also support this well- established view that global measures of impulsivity and self-esteem, but not PSPs, are significantly related to the general impulse buying tendency, and general impulse buying tendency is the strongest predictor of product specific impulse buying tendencies. This suggests that people with a high general impulsive buying tendency are also impulse buyers for different product types and general impulse buying tendency is better explained by measures of impulsivity and self-esteem, indicating a structural (latent) model from global measures of self-system to product specific impulsive buying tendencies through the general impulsivity trait. These general personality measures, however, fail to explain product specific impulse buying tendencies directly, as well as how they change in relation to relevance of the product with the specific aspects of the self. Moreover, the relationship between GSE and impulsive buying disappears after controlling for the effects of self-control and impulsivity. On the other hand, specific self-perceptions such as PSE and body fat were better predictors of product specific impulsive buying tendencies. The observed relationships among physical self-perceptions and product specific impulsive buying tendency were also independent from the effects of self-control and general impulsivity measures.

These preliminary results were also partially supported by some recent previous research reports, e.g., [36] [37] [62]. In their research, Jones and colleagues [62], for example, reported that actual impulse buying behavior is better explained by product specific conceptualizations of impulse buying tendency rather than general measures of impulsivity. Dittmar [17] [36] also emphasized self-related motivations of impulse buying tendency towards certain product categories (i.e. fashionable 
clothing and accessories) among excessive shoppers. Relying on the symbolic self-completion theory, they suggest that perceived discrepancies between the actual and ideal self-images motivate people to consume goods for their symbolic meanings as a compensatory motive. The specific sub-components of the self-system underlying such impulsive buying tendencies, however, remained unexplained.

Study 1 supports and extends previous research on self-related mechanisms of impulsive buying tendencies by showing how impulsive buying tendency towards certain products can be better understood with specific self-perceptions rather than global measures of self-esteem. More importantly, study 1 also confirms the proposed expectation concerning the self-related roles of certain products by implying, for the first time, that the impulsive buying tendency of individuals towards different products may differ depending on the symbolic function of the product in terms of achieving and maintaining specific self-images.

\section{Study 2}

The observed relationships between physical self-esteem and product specific impulsive buying tendencies in study 1 partially supported our initial hypothesis and provided preliminary data on how individuals' impulsive buying tendencies may change depending on the product type. Although these relationships for physical self-esteem were significant, they were, however, weak in general, explaining only $4 \%$ to $11 \%$ of variance in product specific impulsive buying tendencies, and thus were not sufficient to provide convincing evidence. Therefore, Study 2 was mainly designed to further understand the changing nature of impulsive buying tendencies depending on the relevance of the product type to physical self-motives. In this regard, study 2 focused on two selective groups: people with: 1) high; and 2) low physical self-esteem. It was declared that understanding certain psychological characteristic differences between these groups would be helpful to elucidate how impulsive buying tendency may change from one product to another in these selected groups.

In social psychology literature, it has been well documented that people with high physical self-esteem exercise more regularly with health and appearance-related motivations, perceive themselves to be more attractive than others, have high self-presentational confidence, and attribute more importance to physical appearance and more importantly to physical appearance related feedback [50]. However, placing high importance on physical appearance may make these individuals vulnerable to impulsive purchases in situations where they think they can increase the likelihood of receiving positive feedback in social settings regarding their appearance. People with high physical self-esteem will seek situations in which they can maintain their high physical self-esteem and avoid behaviors that may threaten their physical self-images. Regarding impulsive buying contexts, this suggests that people with high physical self-esteem may be more inclined to buy fashionable clothes on impulse since they may expect to get favorable evaluations. These individuals, however, may have more control on their impulsive buying 
tendencies in situations and behaviors that may potentially to distort their physical appearance, such as buying snack foods or binge eating. Moreover, the impulsive buying tendency towards a neutral product in terms of its relevance to physical self-esteem, for example a portable music player, should remain similar regardless of the level of physical self-esteem. Figure 3 summarizes the proposed model for study 2 . In addition to clothing and snack food impulsive buying conditions, technological products (music players) were also used as a neutral condition in study 2 in terms of their relevance to physical self-motives.

Thus, study 2 proposes that the impulsive buying tendency of an individual toward different products will differ depending on the relevance of the product to physical self-motives, and perceived importance differences in the physical domain may be a potential underlying factor in the changing nature of relationships between physical self-esteem and product specific impulsive buying tendencies.

\subsection{Methods}

In study 2, it was specifically aimed to select two skewed groups (high and low PSE) in terms of their physical self-esteem scores. It was considered that those who are at the opposite poles of the distribution in terms of physical self-esteem levels would make the role of physical self-esteem more salient in their purchasing decisions. The first pool of participants was 339 undergraduate and graduate students, from numerous majors such as business, and pre-medical (189 males with Mage $=26.41, \mathrm{SD}=3.17$ and 150 females with Mage $=24.38, \mathrm{SD}=4.20$ ) attending a large US university in the Houston metropolitan area. At the beginning of the study, informed consent forms were distributed describing the general nature of the procedures. The voluntary and confidential nature of the study was emphasized, and students were asked "not" to write their names on any of the questionnaires. Questionnaires were coded with unique ID numbers in advance. Questionnaires were coded with unique ID numbers in advance to protect confidentiality and anonymity. That is, only the investigators can identify

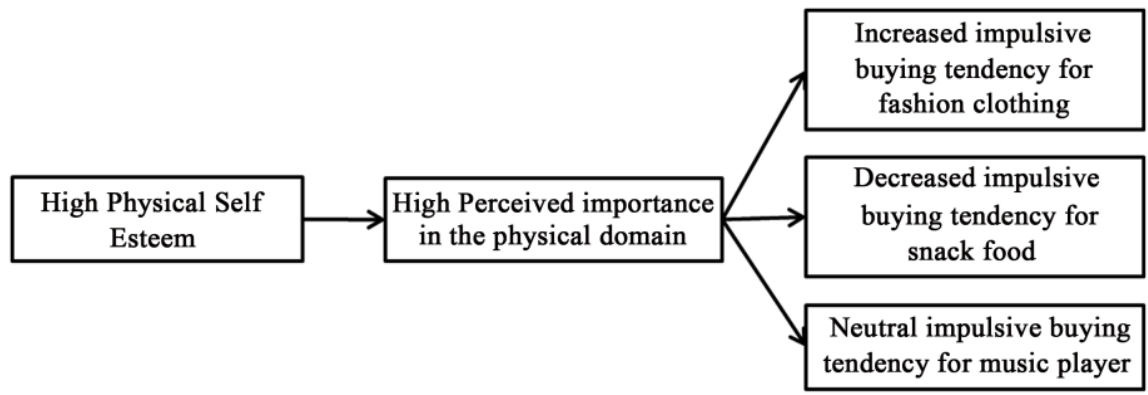

Figure 3. Proposed relationships among variables.

the responses of individual subjects and the confidentiality of information collected from research participants was maintained. Also, the project cannot link individual responses with participants' identities.

The 7 item PSE subscale of the PSDQ, used in study 1, was administered to all 
participants to determine their physical self-esteem level. Purposive sampling procedures were followed to specifically focus on individuals with high and low physical self-esteem. Therefore, each participants' physical self-esteem score was determined right after they completed the PSDQ scale. Participants for the high PSE group were selected among those who had scores of $35-40$ (where the possible range of scores was from 8 to 40) whereas participants for the low PSE group were selected among those who scored 20 and lower in PSE. Based on this selection, however, the number of participants was not equal in each group (51 participants in the high PSE group and 25 participants in the low PSE group). Therefore 25 participants were randomly selected among the high PSE group to finalize grouping.

The selected participants in each group were then asked to complete a modified version of the Perceived Importance Profile (PIP) [67]. The modified PIP allows individuals to assign importance weights to each item in the PSE scale such that a PSE and its perceived importance item are, "I look physically attractive for my age" and "How important is it to you that you look physically attractive for your age".

The modified PIP also consists of 8 items on a 5-point Likert type scale ranging from "very important" to "not important at all".

All participants in each group were also asked to respond to the three different versions of an impulsive buying scenario, originally adopted from [9] that was developed to measure participants' impulse buying decisions for clothing, snack food and music players. The content of each scenario was provided in Table 4. After reading each scenario, participants were asked to indicate their urge and intentions to buy the described product on a 10-point Likert type single item ranging from "I would definitely buy" to "I would definitely NOT buy".

\subsection{Results}

Descriptive (mean and standard deviations) characteristics of all measures are provided in Table 5 both for high PSE and low PSE groups. A series of independent sample t-tests were performed to make preliminary comparisons between high PSE and low PSE groups. Results showed that the high PSE group also has high perceived importance scores in the physical domain $(t(48)=3.94, p<0.05)$. Significant differences on impulsive buying tendencies were also found. The impulsive buying tendency for clothing was significantly higher among individuals with high PSE $(t(48)=2.70, p<0.05)$ whereas individuals with low PSE have a higher tendency to buy snack food on impulse $(t(48)=2.12, p<0.05)$. No significant difference, on the other hand, was found between PSE groups on impulsive buying tendency for the music player $(t(48)=-0.697, p>0.05)$. This supported the argument in our Hypothesis 3 towards people's different Table 4. Impulse buying scenarios.

Conditions Scenario


You have a part-time job and you will get your next paycheck two days from now, and you have only $\$ 50$ left in your debit account. But you also have 2 credit cards you can use. You need to buy a pair of shoes for an outdoor party this weekend. After work, you go to a mall with your friend to buy shoes.

Clothing As you are walking through the mall you see a great looking popular brand shirt on sale for $\$ 60$. You check the size of the shirt and realize it perfectly fits on you. Your friend also gives you a positive comment that you look so good with the shirt. The helpful salesperson tells you that they have just one piece left in this size, and it is unlikely that they will get more pieces in this style in the future. What would be your intention to buy the described shirt from 1 to 10 ?

You have a part-time job and you will get your next paycheck two days from now, and you have only $\$ 50$ left in your debit account. But you also have 2 credit cards you can use. You need to do some grocery shopping. After work you go a market to do grocery. You buy everything you wrote

Snack-Food down earlier; As you are waiting on the cashier line you start feeling hungry and you saw one of your favorite chocolate bars on the shelves next to cashier zone. You check the chocolate bar has 1000 calories which if you eat will make you take extra calories and distort your daily diet. What would be your intention to buy the described product from 1 to 10 ?

You have a part-time job and you will get your next paycheck two days from now, and you have only $\$ 50$ left in your debit account. But you also have 2 credit cards you can use. Your friend asks you to accompany him/her in an electronics store when he/she wants to look for a computer. Although you

Music Player have no intention to buy anything from this store, you see a popular brand portable music player on sale for $\$ 60$. The music player is also coming with one of your favorite bands playlist loaded in it as part of the promotion. The helpful salesperson tells you that they have only few items left for this promotion, and it is unlikely that they will launch such a promotion soon. What would be your intention to buy the described product from 1 to 10 ?

Table 5. Mean and standard deviations of product specific IBT and PSPs between groups.

\begin{tabular}{ccccc}
\hline & \multicolumn{2}{c}{ High PSE } & \multicolumn{2}{c}{ Low PSE } \\
\cline { 2 - 5 } & Mean & Std & Mean & Std \\
\hline Physical Self Esteem & 36.64 & 1.15 & 18.92 & 1.28 \\
Perceived Importance Profile & 33.88 & 3.25 & 30.00 & 3.69 \\
IBT-Clothing & 7.36 & 1.28 & 6.40 & 1.22 \\
IBT-Snack Food & 5.04 & 1.81 & 6.20 & 1.97 \\
IBT-Music Player & 4.76 & 1.45 & 5.08 & 1.77 \\
\hline
\end{tabular}

impulsive buying tendencies of diverse products, depending on the relevance of the product to their physical self-motives.

For main analyses, 2 (Group: High PSE, Low PSE) $\times 3$ (Product type: clothing, snack food, music player) mixed design repeated measure ANOVAs were conducted, both with and without perceived importance as a covariate, to understand: 1) whether impulsive buying tendencies between groups are different as a function of product type; and if so, 2) how this group x product type interaction changes after controlling for the effects of perceived importance on impulsive buying tendencies. A significant multivariate main effect was found for product type 
$\left(\right.$ Wilks' lambda $=0.49, \mathrm{~F}_{(2,47)}=24.57, p=0.000, \eta_{p}^{2}=0.51$, Power $\left.=1.00\right)$ in dicating that when both groups are combined, impulsive buying tendency is significantly different for different product types. Follow up pairwise comparisons with Bonferroni corrections showed that clothing impulsive buying is significantly higher than both snack food $(p=0.002)$ and music player $(p=0.000)$ impulsive buying tendencies. More importantly, a significant multivariate "group $\times$ product type" interaction (Wilks' lambda $=0.81, \mathrm{~F}_{(2,47)}=5.56, p=0.007, \eta_{p}^{2}=0.19$, Power $=0.83$ ) was found, further confirming our hypothesis 3 that impulsive buying tendencies between high PSE and low PSE groups change as a function of product type. Impulsive buying tendencies were plotted in Figure $\mathbf{4}$ for both groups to display the interaction effect. No significant multivariate main effect for "group" was found $(p>0.05)$ showing that when combined, both high PSE and low PSE groups have similar impulsive buying tendencies towards different products.

Perceived importance in the physical domain was used as a covariate in the second set of analyses to examine how the "group $\times$ product type" interaction changes after controlling for the effects of PIP scores. No significant multivariate main effect was found for both product type $(p>0.05)$ and group $(p>0.05)$ indicating that on average the impulsive buying tendency does not change among product types and between groups, respectively. On the other hand, Group $\times$ product type interaction was significant (Wilks' lambda $=0.87, \mathrm{~F}_{(2,47)}=3.27, p=$ $0.047, \eta_{p}^{2}=0.12$, Power $=0.59$ ) indicating that impulsive buying tendency differences between high PSE and low PSE groups change as a function of product type even after controlling for the effects of PIP.

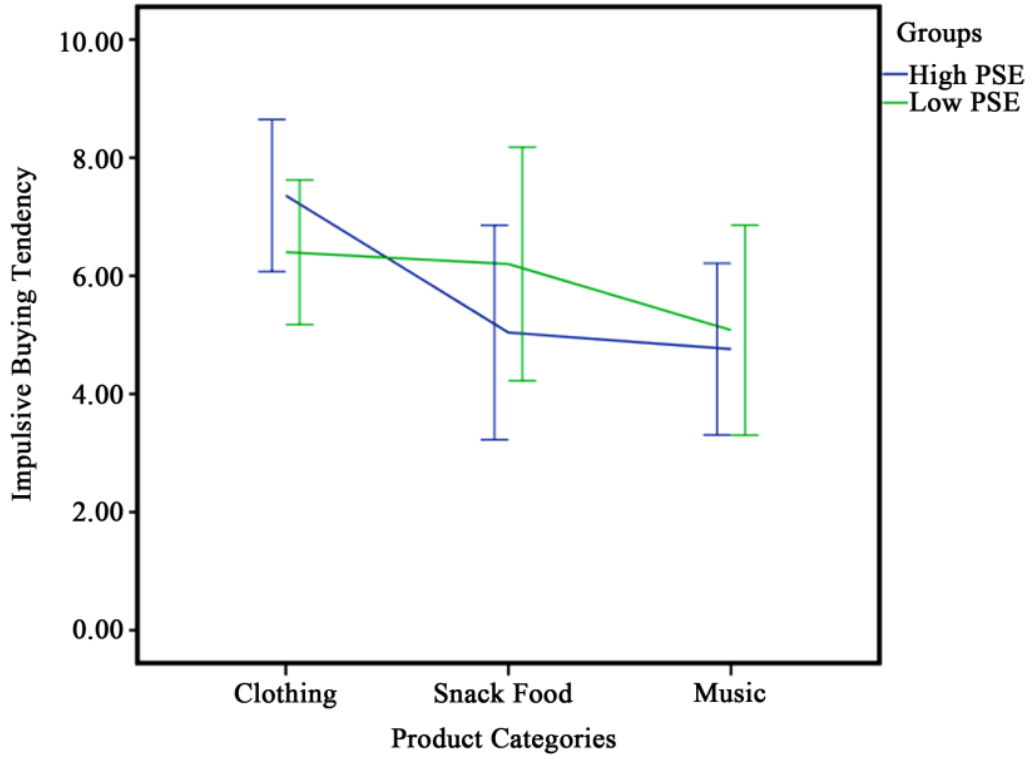

Figure 4. Impulse buying tendency for different products for high PSE and Low PSE groups.

However, comparing the statistical parameters of two analyses showed that, when PIP is used as a covariate, $p$-values increase ( $p=0.007$ vs $p=0.047)$, and both effect size $\left(\eta_{p}^{2}=0.19\right.$ vs $\left.\eta_{p}^{2}=0.12\right)$ and power ( 0.83 vs 0.59$)$ decreases 
considerably, suggesting a partial effect of PIP on impulsive buying tendencies.

\subsection{Study 2 Discussion}

Study 2 was designed to further investigate the changing nature of impulsive buying tendencies depending on the relevance of products to physical self-motives. Individuals with high and low physical self-esteem were specifically selected and grouped to examine role of PSE on buying tendencies. Preliminary analyses of the data from study 2 further confirmed the primary propositions of the current research that, in addition to be a general personality characteristic, impulsive buying is also a product specific tendency regardless of PSE level. Compared to impulsive buying levels for snack food and the music player, a higher impulsive buying tendency for clothing in both groups suggests that certain products are more likely to be bought on impulse than others. In a series of studies, Ditmarr and colleagues [17] [18] [36] [42] also consistently showed products closely related to self-image and self- presentation, such as fashion clothing, jewelry, and accessories, are reported to be bought more on impulse than other products as a coping strategy to reduce the perceived gap between desired and actual self-images. The current study also supports and further extends support Dittmar and colleagues' findings by showing that physical self-esteem levels, along with perceived importance of the physical domain, may change the nature of the impulse buying tendency of the individual depending on the relevance of the product to physical self-motive. However, apart from previous interpretations, current results also suggest that people who have impulsive buying tendencies for certain products may not necessarily be motivated by impaired or wounded self-images in a "reactive" manner but may also be motivated by self-protective reasons in a "pro-active" way to maintain their already enhanced self-images in future social settings.

Results from study 1 and study 2 imply a product-specific cognitive evaluation component even in impulsive purchases based on both how individuals perceive themselves physically and the relevance of specified products to their physical image. Impulsive buying behavior, however, has traditionally been characterized as a general tendency to buy in a spontaneous, unplanned and thoughtless way. High impulsive buyers are assumed to act unreflectively in shopping realms. Therefore, current results, at the first glance, may seem to conflict with the traditional conceptualizations of impulsive buying since it suggests self-image related cognitions as moderating factors in the relationship between certain products and impulsive buying tendencies. This traditional understanding, however, is undermined and questioned by some prominent studies. For example, Rook and Fisher [9] also examined how individuals' normative evaluations regarding purchase decisions change the strength of the relationship between impulse buying tendency and subsequent impulse buying behavior. They reported that individuals' tendency to buy on impulse is not correlated with their actual impulse buying decisions if they have negative normative evaluations towards the buying context [9]. In accordance with Dittmar and colleagues propositions in [17] [18] [36] [42], these results also suggest that although impulsive buying tendency of the individual is a main 
motivator for actual impulse buying behaviors, various factors such as economic position of the person, normative evaluations regarding buying context, social visibility of impulsive buying and symbolic meanings of the products may potentially influence impulsive buying behaviors. In this regard, physical-self perceptions can also be considered as one of the subjective norms that may modulate purchasing decisions depending on the buying context. The perceived function or relevance of the specified product to the currently held physical self-images may change the level of impulsive buying tendency and, thus, may impact whether the product will be bought on impulse or not.

Study 2 also provides some clear insights regarding underlying factors for the role of physical self-perception on the nature of impulsive buying tendencies. People with high physical self-esteem, for example, also reported that they attribute importance to their physical characteristics, abilities and the impressions they made on others. Results indicated that when this perceived importance in the physical domain is accompanied by high physical self-esteem it may create a specific impulsive buying profile that reacts selectively to different items as a function of their relevance to physical-self-images. In the snack-food scenario, for example, since people with high PSE have also reported high PIP they may consider unplanned and excessive snack food consumption as a potential future threat to distort their physical appearance and thus may increase the probability of getting negative evaluations in the physical domain. Although product specific impulsive buying tendencies were significantly different between high PSE and low PSE groups, the magnitude of significance considerably diminished after controlling for the effect of perceived importance in the physical domain, suggesting a partial effect of perceived importance for the role of physical self-esteem level on product-specific impulsive buying tendencies.

Despite consistent findings, the current results should be considered cautiously due to some limitations of the study. The unrepresentative nature of the sample restricts the generalizability of the results since both study 1 and study 2 were mostly conducted with undergrad level university students. The sample size is relatively small in study 2 and restricted to specifically selected groups, thus making it difficult to extend findings to other populations. In addition, correlational analyses with cross sectional design does not allow one to establish solid causational interpretations for the role of physical self-perceptions on impulsive buying tendencies. Moreover, current studies did not attempt model testing, which could provide more clear insights regarding the underlying mechanisms of observed relationships. Rather, it was primarily designed to explore relationships between domain specific self-perceptions and product specific impulsive buying tendencies and confirm these preliminary findings in specifically selected groups. Further studies, therefore, should be conducted with larger sample sizes in selected groups and employ structural equation modeling (SEM) to further elucidate the underlying mechanisms by which the physical self-esteem level of the individual changes the nature of impulsive buying tendencies. Qualitative research designs can also be integrated with quantitative methods to better understand individual factors affecting why people 
who have high PSE and PIP are impulsive buyers for certain products but not others.

\section{General Conclusions}

Impulsive buying is a complex behavior that occurs because of the interactions among various psychological, social and personality factors. General trait approaches, relating impulsive buying behavior primarily to the general impulsiveness level of the individual and assuming that individuals are impulsive buyers for all products, still explain most of the variance in the impulsive buying tendency but are not adequate to explain the product-specific nature of impulsive buying tendencies and how these tendencies are changing because of the symbolic and psychological functions of the products. Taken together, the present results indicated that physical self-images and perceived importance attributed to these images should also be considered as perceptual variables with the potential to change impulsive buying tendencies in a context-specific manner.

\section{References}

[1] Ajzen, I. (1991) The Theory of Planned Behavior. Organizational Behavior and Human Decision Processes, 50, 179-211. https://doi.org/10.1016/0749-5978(91)90020-T

[2] Becker, G.S. (2013) The Economic Approach to Human Behavior. University of Chicago Press.

[3] Kirchgässner, G. (2008) Homo Oeconomicus: The Economic Model of Behaviour and Its Applications in Economics and Other Social Sciences (Vol. 6). Springer Science \& Business Media.

[4] Ajzen, I. and Fishbein, M. (1977) Attitude-Behavior Relations-Theoretical-Analysis and Review of Empirical-Research. Psychological Bulletin, 84, 888-918. https://doi.org/10.1037/0033-2909.84.5.888

[5] Ajzen, I., Timko, C. and White, J.B. (1982) Self-Monitoring and the Attitude Behavior Relation. Journal of Personality and Social Psychology, 42, 426-435. https://doi.org/10.1037/0022-3514.42.3.426

[6] Baumeister, R.F. (2002) Yielding to Temptation: Self-Control Failure, Impulsive Purchasing, and Consumer Behavior. Journal of Consumer Research, 28, 670-676. https://doi.org/10.1086/338209

[7] Beatty, S.E. and Ferrell, M.E. (1998) Impulse Buying: Modeling Its Precursors. Journal of Retailing, 74, 169-191. https://doi.org/10.1016/S0022-4359(99)80092-X

[8] Luna-Arocas, R. (2008) Self-Discrepancy and Impulse Buying: An Exploratory Study. International Journal of Organization Theory and Behavior, 11, 240.

[9] Rook, D.W. and Fisher, R.J. (1995) Normative Influences on Impulsive Buying Behavior. Journal of Consumer Research, 22, 305-313. https://doi.org/10.1086/209452

[10] Kossman, S. (2016) Survey: 5 in 6 Americans Admit to Impulse Buys. https://www.creditcards.com/credit-card-news/impulse-buy-survey.php

[11] Hausman, A. (2000) A Multi-Method Investigation of Consumer Motives in Impulse Buying Behavior. Journal of Consumer Marketing, 17, 403-419. https://doi.org/10.1108/07363760010341045

[12] Inman, J.J. and Winer, R.S. (1998) Where the Rubber Meets the Road: A Model of In-Store Consumer Decision Making.

[13] Kacen, J.J., Hess, J.D. and Walker, D. (2012) Spontaneous Selection: The Influence of Product and Retailing Factors on Consumer Impulse Purchases. Journal of Retailing and Consumer Services, 19, 578-588. https://doi.org/10.1016/j.jretconser.2012.07.003 
[14] Mattila, A.S. and Wirtz, J. (2008) The Role of Store Environmental Stimulation and Social Factors on Impulse Purchasing. Journal of Services Marketing, 22, 562-567. https://doi.org/10.1108/08876040810909686

[15] West, J.C. (1951) Results of Two Years' of Study into Impulse Buying. Journal of Marketing, 15, 362-363. https://doi.org/10.2307/1247377

[16] Black, D.W. (2007) A Review of Compulsive Buying Disorder. World Psychiatry, 6, 14-18.

[17] Dittmar, H., Beattie, J. and Friese, S. (1995) Gender Identity and Material Symbols: Objects and Decision Considerations in Impulse Purchases. Journal of Economic Psychology, 16, 491-511. https://doi.org/10.1016/0167-4870(95)00023-H

[18] Dittmar, H., Beattie, J. and Friese, S. (1996) Objects, Decision Considerations and Self-Image in Men's and Women's Impulse Purchases. Acta Psychologica, 93, 187-206. https://doi.org/10.1016/0001-6918(96)00019-4

[19] Faber, R.J. and Vohs, K.D. (2004) To Buy or Not to Buy? Self-Control and Self-Regulatory Failure in Purchase Behavior. In R. F. Baumeister, \& K. D. Vohs (Eds.), Handbook of Self-Regulation: Research, Theory, and Applications (pp. 509-524). Guilford, New York.

[20] Verplanken, B. and Sato, A. (2011) The Psychology of Impulse Buying: An Integrative Self-Regulation Approach. Journal of Consumer Policy, 34, 197-210. https://doi.org/10.1007/s10603-011-9158-5

[21] Verplanken, B., Herabadi, A.G., Perry, J.A. and Silvera, D.H. (2005) Consumer Style and Health: The Role of Impulsive Buying in Unhealthy Eating. Psychology \& Health, 20, 429-441. https://doi.org/10.1080/08870440412331337084

[22] Rook, D.W. (1987) The Buying Impulse. Journal of Consumer Research, 14, 189-199. https://doi.org/10.1086/209105

[23] Kacen, J.J. and Lee, J.A. (2002) The Influence of Culture on Consumer Impulsive Buying Behavior. Journal of Consumer Psychology, 12, 163-176. https://doi.org/10.1207/S15327663JCP1202_08

[24] Stern, H. (1962) The Significance of Impulse Buying Today. Journal of Marketing, 26, 59-62. https://doi.org/10.2307/1248439

[25] Clover, V.T. (1950) Relative Importance of Impulse-Buying in Retail Stores. Journal of Marketing, 15, 66-70. https://doi.org/10.2307/1247083

[26] Pentecost, R. and Andrews, L. (2010) Fashion Retailing and the Bottom Line: The Effects of Generational Cohorts, Gender, Fashion Fanship, Attitudes and Impulse Buying on Fashion Expenditure. Journal of Retailing and Consumer Services, 17, 43-52. https://doi.org/10.1016/j.jretconser.2009.09.003

[27] Puri, R. (1996) Measuring and Modifying Consumer Impulsiveness: A Cost-Benefit Accessibility Framework. Journal of Consumer Psychology, 5, 87-113. https://doi.org/10.1207/s15327663jcp0502_01

[28] Cox, K. (1964) The Responsiveness of Food Sales to Self-Space Changes in Supermarkets. Journal of Marketing Research, 63-67. https://doi.org/10.2307/3149924

[29] Patterson, L.W. (1963) In-Store Traffic Flow. Point-of-Purchasing Advertising Institute, New York.

[30] Kollat, D.T. and Willett, R.P. (1967) Consumer Impulse Purchasing Behavior. Journal of Marketing Research, 4, 21-31. https://doi.org/10.2307/3150160

[31] Shapiro, I.J. (1973) Marketing Terms: Definitions. Explanations and/or Aspects. 3rd Edition, West Long Branch, S-M-C.

[32] Ainslie, G. (1975) Specious Reward: A Behavioral Theory of Impulsiveness and Impulse Control. Psychological Bulletin, 82, 463-509. https://doi.org/10.1037/h0076860

[33] Dittmar, H. (1992) The Social Psychology of Material Possessions: To Have Is to Be. Hemel Hampstead, Harvester Wheatsheaf and St Martin's Press, New York.

[34] Wood, M. (1998) Socio-Economic Status, Delay of Gratification, and Impulse Buying. Journal of Economic Psychology, 19, 295-320. https://doi.org/10.1016/S0167-4870(98)00009-9

[35] Amos, C., Holmes, G.R. and Keneson, W.C. (2014) A Meta-Analysis of Consumer 
Impulse Buying. Journal of Retailing and Consumer Services, 21, 86-97. https://doi.org/10.1016/j.jretconser.2013.11.004

[36] Dittmar, H. and Drury, J. (2000) Self-Imagine-Is It in the Bag? A Qualitative Comparison between "Ordinary" and "Excessive" Consumers. Journal of Economic Psychology, 21, 109-142. https://doi.org/10.1016/S0167-4870(99)00039-2

[37] Lucas, M. and Koff, E. (2014) The Role of Impulsivity and of Self-Perceived Attractiveness in Impulse Buying in Women. Personality and Individual Differences, 56, 111-115. https://doi.org/10.1016/j.paid.2013.08.032

[38] Silvera, D.H., Lavack, A.M. and Kropp, F. (2008) Impulse Buying: The Role of Affect, Social Influence, and Subjective Wellbeing. Journal of Consumer Marketing, 25, 23-33. https://doi.org/10.1108/07363760810845381

[39] Sultan, A.J., Joireman, J. and Sprott, D.E. (2011) Building Consumer Self-Control: The Effect of Self-Control Exercises on Impulse Buying Urges. Marketing Letters, 23, 61-72. https://doi.org/10.1007/s11002-011-9135-4

[40] Youn, S. and Faber, R.J. (2000) Impulse Buying: Its Relation to Personality Traits and Cues. Advances in Consumer Research, 27, 179-185.

[41] Herabadi, A.G., Verplanken, B. and van Knippenberg, A. (2009) Consumption Experience of Impulsive Buying in Indonesia: Emotional Arousal and Hedonistic Considerations. Asian Journal of Social Psychology, 12, 20-31. https://doi.org/10.1111/j.1467-839X.2008.01266.x

[42] Dittmar, H. (2005) A New Look at "Compulsive Buying": Self Discrepancy and Materialistic Values as Predictors of Compulsive Buying Tendency. Journal of Social and Clinical Psychology, 24, 832-859. https://doi.org/10.1521/jscp.2005.24.6.832

[43] Dittmar, H. and Bond, R. (2010) "I Want It and I Want It Now": Using a Temporal Discounting Paradigm to Examine Predictors of Consumer Impulsivity. The British Journal of Social Psychology, 101, 751-776. https://doi.org/10.1348/000712609X484658

[44] Sivanathan, N. and Pettit, N.C. (2010) Protecting the Self through Consumption: Status Goods as Affirmational Commodities. Journal of Experimental Social Psychology, 46, 564-570. https://doi.org/10.1016/j.jesp.2010.01.006

[45] Wicklund, R.A. and Gollwitzer, P.M. (1982) Symbolic Self-Completion. Erlbaum, Hillsdale.

[46] Higgins, T. (1987) Self-Discrepancy: A Theory Relating Self to Affect. Psychological Review, 94, 319-340. https://doi.org/10.1037/0033-295X.94.3.319

[47] Fox, K.R. (1997) The Physical Self and Processes in Self-Esteem Development. In: Fox, K.R., Ed., The Physical Self: From Motivation to Well-Being, Human Kinetics, Champaign, 111-141.

[48] Asci, F.H. (2004) Physical Self-Perception of Elite Athletes and Non-Athletes: A Turkish Sample. Perceptual and Motor Skills, 99, 1047-1052. https://doi.org/10.2466/pms.99.3.1047-1052

[49] Asci, F.H., Kin, A. and Kosar, N. (1998) Effect of Participation in an 8 Week Aerobic Dance and Step Dance Aerobics Program on Physical Self-Perception and Body Image Satisfaction. International Journal of Sport Psychology, 29, 366-375.

[50] Fox, K.R. (2000) self-Esteem, Self-Perceptions and Exercise. International Journal of Sport Psychology, 31, 228-240.

[51] Lindwall, M. and Hassmen, P. (2004) The Role of Exercise and Gender for Physical Self-Perceptions and Importance Ratings in Swedish University Students. Scandinavian Journal of Medicine and Science in Sports, 14, 373-380. https://doi.org/10.1046/j.1600-0838.2003.372.x

[52] Shavelson, R.J., Hubner, J.J. and Stanton, G.C. (1976) Self-Concept: Validation of Construct Interpretations. Review of Educational Research, 46, 407-441. https://doi.org/10.3102/00346543046003407

[53] Brown, J.D. (1998) The Self. McGraw-Hill, New York.

[54] Marsh, H.W. (1990) A Multidimensional, Hierarchical Model of Self-Concept: Theoretical and Empirical Justification. Educational Psychology Review, 2, 77-172. https://doi.org/10.1007/BF01322177

[55] Sonstroem, R.J. (1997) The Physical Self-System: A Mediator of Exercise and 
Self-Esteem. In: Fox, K.R., Ed., The Physical Self: From Motivation to Well-Being, Human Kinetics, Champaign, 3-26.

[56] Shiling, C. (1993) The Body and Social Theory. Sage, London.

[57] Giddens, A. (1991) Modernity and Self-Identity. Polity, Cambridge.

[58] Synnott, A. (1993) The Body Social: Symbolism, Self and Society. Routledge, London and New York.

[59] Baumeister, R.F. (1998) The Self. In: Gilbert, D.T., Fiske, S.T. and Lindzey, G., Eds., Handbook of Social Psychology, McGraw-Hill, New York, 4th Edition, 680-740.

[60] Baumeister, R.F. (2005) The Self. In: Baumeister, R.F. and Finkel, E.F., Eds., Advanced Social Psychology: The State of the Science, Oxford, New York, 139-176.

[61] Kirk, D. (1993) The Body, Schooling and Culture. Deakin University Press, Victoria.

[62] Jones, M.A., Reynolds, K.E., Weun, S. and Beatty, S.E. (2003) The Product-Specific Nature of Impulse Buying Tendency. Journal of Business Research, 56, 505-511. https://doi.org/10.1016/S0148-2963(01)00250-8

[63] Rosenberg, M. (1979) Conceiving the Self. Basic Books, New York.

[64] Marsh, H.W., Richards, G.E., Johnson, S., Roche, L. and Tremayne, P. (1994) Physical Self-Description Questionnaire: Psychometric Properties and a Multitrait-Multimethod Analysis of Relations to Existing Instruments. Journal of Sport and Exercise Psychology, 16, 270-305. https://doi.org/10.1123/jsep.16.3.270

[65] Tangney, J.P., Baumeister, R.F. and Boone, A.L. (2004) High Self-Control Predicts Good Adjustment, Less Pathology, Better Grades, and Interpersonal Success. Journal of Personality, 72, 271-324. https://doi.org/10.1111/j.0022-3506.2004.00263.x

[66] Tellegen, A. (1982) Brief Manual for the Multidimensional Personality Questionnaire. Unpublished Manuscripts, University of Minnesota, Minneapolis, 1031-1010.

[67] Lindwall, M., Aşçi, F.H., Palmeira, A., Fox, K.R. and Hagger, M.S. (2011) The Importance of Importance in the Physical Self: Support for the Theoretically Appealing But Empirically Elusive Model of James. Journal of Personality, 79, 303-334. https://doi.org/10.1111/j.1467-6494.2010.00678.x 